\title{
Well-Pointed Coalgebras (Extended Abstract) ${ }^{\star}$
}

\author{
Jiří Adámek ${ }^{1}$, Stefan Milius ${ }^{1}$, Lawrence S. Moss ${ }^{2}$, and Lurdes Sousa ${ }^{3, \star \star}$ \\ 1 Institut für Theoretische Informatik, \\ Technische Universität Braunschweig, Germany \\ adamek@iti.cs.tu-bs.de, mail@stefan-milius.eu \\ 2 Department of Mathematics, Indiana University, Bloomington, IN, USA \\ lsm@indiana.edu \\ 3 Departamento de Matemática, Instituto Politécnico de Viseu, Portugal \\ sousa@mat.estv.ipv.pt
}

\begin{abstract}
For set functors preserving intersections, a new description of the final coalgebra and the initial algebra is presented: the former consists of all well-pointed coalgebras. These are the pointed coalgebras having no proper subobject and no proper quotient. And the initial algebra consists of all well-pointed coalgebras that are well-founded in the sense of Taylor 16]. Finally, the initial iterative algebra consists of all finite well-pointed coalgebras. Numerous examples are discussed e.g. automata, graphs, and labeled transition systems.
\end{abstract}

\section{Introduction}

Initial algebras are known to be of primary interest in denotational semantics, where abstract data types are often presented as initial algebras for an endofunctor $H$ expressing the type of the constructor operations of the data type. For example, finite binary trees are the initial algebra for the functor $H X=X \times X+1$ on sets. Analogously, final coalgebras for an endofunctor $H$ play an important role in the theory of systems developed by Rutten [13]: $H$ expresses the system type, i. e., which kind of one-step reactions states can exhibit (input, output, state transitions etc.), and the elements of a final coalgebra represent the behavior of all states in all systems of type $H$ (and the unique homomorphism from a system into the final one assign to every state its behavior). For example, deterministic automata with input alphabet $I$ are coalgebras for $H X=X^{I} \times\{0,1\}$, the final coalgebra is the set of all languages on $I$.

In this paper a unified description is presented for (a) initial algebras, (b) final coalgebras and (c) initial iterative algebras (in the automata example this is the set of all regular languages on $I$ ). We also demonstrate that this new description

\footnotetext{
* The full version containing all proofs is available at http://www.iti.cs.tu-bs.de/TI-INFO/milius/research/wells.full.pdf

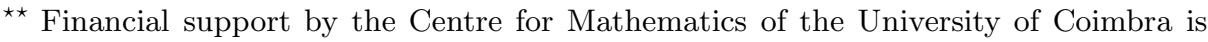
acknowledged.
} 
provides a unifying view of a number of other important examples. We work with set functors $H$ preserving intersections. This is a requirement that many "everyday" set functors satisfy. We prove that the final coalgebra of $H$ can then be described as the set of all well-pointed coalgebras, i.e., pointed coalgebras not having any proper subobject and also not having any proper quotient. Moreover, the initial algebra can be described as the set of all well-pointed coalgebras which are well-founded in the sense of Taylor [16. A coalgebra $(A, \alpha)$ is well-founded if no proper subcoalgebra $\left(A^{\prime}, \alpha^{\prime}\right)$ of $(A, \alpha)$ forms a pullback

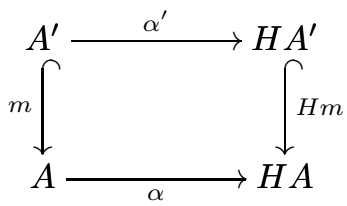

This concept was first studied by Osius [12] for graphs considered as coalgebras of the power-set functor $\mathscr{P}$ : a graph is well-founded iff it has no infinite paths. Taylor [1617] introduced well-founded coalgebras for general endofunctors, and he proved that for endofunctors preserving inverse images the concepts of initial algebra and final well-founded coalgebra coincide.

We are going to prove that this result holds for every set functor $H$; the step towards making no assumptions on $\mathrm{H}$ is non-trivial. And if $H$ preserves intersections, we describe its final coalgebra, initial algebra, and initial iterative algebra using well-pointed coalgebras as above. The first result will be proved in a much more general context, working with an endofunctor of a locally finitely presentable category preserving finite intersections, but this extra assumption can be dropped in the case of set functors.

\section{Well-Founded Coalgebras}

Throughout this section $\mathscr{A}$ denotes an LFP category with a simple initial object. And $H$ is an endofunctor preserving monomorphisms. Let us recall these concepts:

Definition 2.1. 1. A category $\mathscr{A}$ is locally finitely presentable (LFP) if

(a) $\mathscr{A}$ is complete, and

(b) there is a set of finitely presentable objects whose closure under filtered colimits is all of $\mathscr{A}$.

2. An object $A$ is called simple if it has no proper quotients. That is, every epimorphism with domain $A$ is invertible.

Example 2.2. The categories of sets, graphs, posets, and semigroups are locally finitely presentable. The initial objects of these categories are empty, hence simple. The category of rings is LFP but the initial object $\mathbb{Z}$ is not simple.

Notation 2.3. For every endofunctor $H$ denote by Coalg $H$ the category of coalgebras $\alpha: A \longrightarrow H A$ and coalgebra homomorphisms, where a homomorphism $h$ from $(A, \alpha)$ to $(B, \beta)$ is a morphism $h: A \longrightarrow B$ such that $\beta \cdot h=H h \cdot \alpha$. 
Remark 2.4. There are some consequences of the LFP assumption that play an important role in our development. These pertain to monomorphisms.

1. $\mathscr{A}$ has (strong epi, mono)-factorizations; see 1.16 in [5]. Recall that an epimorphis is strong iff it has the diagonal fill-in property w.r.t. all monomorphisms.

2. $\mathscr{A}$ is wellpowered, see 1.56 in [5]. This implies that for every object $A$ the poset $\operatorname{Sub}(A)$ of all subobjects of $A$ is a complete lattice.

3. Monomorphisms are closed under filtered colimits; see 1.62 in [5].

Since subcoalgebras play a basic role in the whole paper, and quotients are important from Section 3 onwards, we need to make clear what we mean by those. We use the term subcoalgebra of a coalgebra $(A, \alpha)$ to mean a subobject $m:\left(A^{\prime}, \alpha^{\prime}\right) \longrightarrow(A, \alpha)$ represented by a monomorphism $m$ in $\mathscr{A}$. Then $m$ is clearly a monomorphism of Coalg $H$; however, in general, monomorphisms in Coalg $H$ need not be carried by monomorphisms from $\mathscr{A}$. As usual, if a subcoalgebra $m$ is not invertible, it is said to be proper. What about quotient coalgebras? A quotient of a coalgebra $(A, \alpha)$ is represented by $e:(A, \alpha) \longrightarrow\left(A^{\prime}, \alpha^{\prime}\right)$, where $e$ is a strong epimorphism in $\mathscr{A}$. Since $H$ is assumed to preserve monomorphisms, Coalg $H$ has factorizations of morphisms $f:(A, \alpha) \longrightarrow(B, \beta)$ into homomorphisms $e:(A, \alpha) \longrightarrow(C, \gamma)$ and $m:(C, \gamma) \longrightarrow(B, \beta)$, i. e., such that $(C, \gamma)$ is a quotient of $(A, \alpha)$ and a subcoalgebra of $(B, \beta)$.

Definition 2.5. A cartesian subcoalgebra of a coalgebra $(A, \alpha)$ is a subcoalgebra $\left(A^{\prime}, \alpha^{\prime}\right)$ forming a pullback (1.1). A coalgebra is called well-founded if it has no proper cartesian subcoalgebra.

Example 2.6. (1) The concept of well-founded coalgebra was introduced originally by Osius [12] for the power set functor $\mathscr{P}$. A graph is a coalgebra $(A, a)$ for $\mathscr{P}$, where $a(x)$ is the set of neighbors of $x$ in the graph. Then a subcoalgebra of $A$ is an (induced) subgraph $A^{\prime}$ with the property that every neighbor of a vertex of $A^{\prime}$ lies in $A^{\prime}$. The subgraph $A^{\prime}$ is cartesian iff it contains every vertex all of whose neighbors lie in $A^{\prime}$. The graph $A$ is a well-founded coalgebra iff it has no infinite path.

(2) Let $A$ be a deterministic automaton considered as a coalgebra for $H X=$ $X^{I} \times\{0,1\}$. A subcoalgebra $A^{\prime}$ is cartesian iff it contains every state all whose successors (under the inputs from $I$ ) lie in $A^{\prime}$. This holds, in particular, for $A^{\prime}=\emptyset$. Thus, no nonempty automaton is well-founded.

(3) Coalgebras for $H X=X+1$ are dynamical systems with deadlocks, see [13. A subcoalgebra $A^{\prime}$ of a dynamical system $A$ is cartesian iff it contains all deadlocks and every state whose next state lies in $A^{\prime}$.

A dynamical system is well-founded iff it has no infinite computation.

Definition 2.7. Every coalgebra $\alpha: A \longrightarrow H A$ induces an endofunction of $\operatorname{Sub}(A)$ (see Remark 2.4.2) assigning to a subobject $m: A^{\prime} \longrightarrow A$ the inverse image $\bigcirc m$ of $H m$ under $\alpha$, i. e., we have a pullback square: 


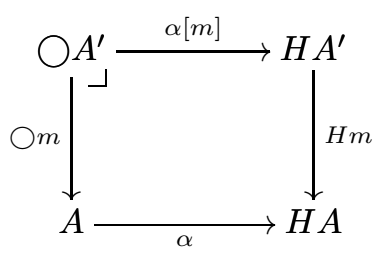

This function $m \longmapsto \bigcirc m$ is obviously order-preserving. By the Knaster-Tarski fixed point theorem, this function has a least fixed point.

Incidentally, the notation $\bigcirc m$ comes from modal logic, especially the areas of temporal logic where one reads $\bigcirc \phi$ as " $\phi$ is true in the next moment," or "next time $\phi "$ for short.

Example 2.8. Recall our discussion of graphs from Example 2.6(1). The pullback $\bigcirc A^{\prime}$ of a subgraph $A^{\prime}$ is the set of vertices of $A$ all of whose neighbors belong to $A^{\prime}$.

Remark 2.9. As we mentioned in the introduction, the concept of well-founded coalgebra was introduced by Taylor [1617. Our formulation is a bit simpler. In [17. Definition 6.3.2] he calls a coalgebra $(A, \alpha)$ well-founded if for every pair of monomorphisms $m: U \longrightarrow A$ and $h: H \longrightarrow U$ such that $h \cdot m$ is the inverse image of $H m$ under $\alpha$ it follows that $m$ is an isomorphism. Thus in lieu of fixed points of $m \longmapsto \bigcirc m$ he uses pre-fixed points.

In addition, our overall work has a methodological difference from Taylor's that is worth mentioning at this point. Taylor is giving a general account of recursion and induction, and so he is concerned with general principles that underlie these phenomena. Indeed, he is interested in settings like non-boolean toposes where classical reasoning is not necessarily valid. On the other hand, in this paper we are studying initial algebras, final coalgebras, and similar concepts, using standard classical mathematical reasoning. In particular, we make free use of transfinite recursion. The definitions in Notation 2.10 just below would look out of place in Taylor's paper. But we believe they are an important step in our development.

Notation 2.10. (a) For every coalgebra $\alpha: A \longrightarrow H A$ denote by

$$
a^{*}: A^{*} \longrightarrow A
$$

the least fixed point of the function $m \longmapsto \bigcirc m$ of Definition 2.7. (Thus, $(A, a)$ is well-founded iff $a^{*}$ is invertible.) Since $a^{*}$ is a fixed point we have a coalgebra structure $\alpha^{*}: A^{*} \longrightarrow H A^{*}$ making $a^{*}$ a coalgebra homomorphism.

(b) For every coalgebra $a: A \longrightarrow H A$ we define a chain of subobjects

$$
a_{i}^{*}: A_{i}^{*} \longrightarrow A \quad(i \in \mathbf{O r d})
$$

of $A$ in $\mathscr{A}$ by transfinite recursion: $a_{0}^{*}: 0 \longrightarrow A$ is the unique morphism; $a_{i+1}^{*}=\bigcirc a_{i}^{*}$ and for limit ordinals $a_{i}^{*}=\bigcup_{j<i} a_{j}^{*}$. Since 0 is simple, $a_{0}^{*}$ is a 
monomorphisms. Moreover, what we have is nothing else than the construction of the least fixed point of $m \longmapsto \bigcirc m$ (cf. Remark 2.9) in the proof of the Knaster-Tarski Theorem in [15. Thus, $a^{*}=\bigcup_{i \in \mathbf{O r d}} a_{i}^{*}$. Also, there exists an ordinal $i$ with $A^{*}=A_{i}^{*}$ (due to wellpoweredness). Henceforth, we call $A^{*}$ the smallest cartesian subcoalgebra of $A$.

Proposition 2.11. For every coalgebra $(A, \alpha)$, the smallest cartesian subcoalgebra $\left(A^{*}, \alpha^{*}\right)$ is its coreflection in the full subcategory of well-founded coalgebras.

Remark. We thus prove that $\left(A^{*}, \alpha^{*}\right)$ is well-founded, and for every homomorphism $f:(B, \beta) \longrightarrow(A, \alpha)$ with $(B, \beta)$ well-founded there exists a unique homomorphism $\bar{f}:(B, \beta) \longrightarrow\left(A^{*}, \alpha^{*}\right)$ with $f=a^{*} \cdot \bar{f}$.

Corollary 2.12. The full subcategory of Coalg $H$ consisting of the well-founded coalgebras is closed under quotients and colimits in Coalg $H$.

For endofunctors preserving inverse images the above corollary is Exercise VI.16 in Taylor [17] and the following theorem is Corollary 9.9 of [16]. As we mentioned in the introduction, it is non-trivial to relax the assumption on the endofunctor, and so our proof is different from Taylor's.

Theorem 2.13. If $H$ preserves finite intersections, then

initial algebra $=$ final well-founded coalgebra.

That is, an algebra $\varphi: H I \longrightarrow I$ is initial iff $\varphi^{-1}: I \longrightarrow H I$ is the final well-founded coalgebra.

Proof (Sketch). (a) Let $I$ be an initial algebra. It follows from [20] that $I$ is obtained as $H^{i} 0$ for some ordinal $i$ for the initial chain introduced in 2 defined by $H^{0} 0=0, H^{i+1} 0=H\left(H^{i} 0\right)$ and $H^{i} 0=\operatorname{colim}_{j<i} H^{j} 0$ for limit ordinals $i$. We prove by transfinite induction that if $I=H^{k} 0$ then the connecting morphisms $H^{i} 0 \longrightarrow H^{k} 0$ for $i \leq k$ are precisely $a_{i}^{*}$ of Notation 2.10. Consequently, $I$ is well-founded. We next use the concept of recursive coalgebra of Capretta et al [6]: It is a coalgebra from which a unique coalgebra-to-algebra morphism into every algebra exists. Initial algebras are proved there to be precisely the final recursive coalgebras. We prove that every well-founded coalgebra is recursive. We thus derive that $I$ is a final well-founded coalgebra.

(b) Let $\psi: I \longrightarrow H I$ be a final well-founded coalgebra. Factorize $\psi=m \cdot e$ where $e$ is a strong epimorphism and $m$ a monomorphism (Remark 2.4). By diagonal fill-in we obtain a quotient $e:(I, \psi) \longrightarrow\left(I^{\prime}, \psi^{\prime}\right)$ which, by Corollary 2.12, is well-founded, thus recursive. Consequently, a coalgebra homomorphism $f:\left(I^{\prime}, \psi^{\prime}\right) \longrightarrow(I, \psi)$ exists. Then $f \cdot e$ is an endomorphism of the final well-founded coalgebra, hence, $f \cdot e=\mathrm{id}_{I}$. This proves that $e$ is an isomorphism, thus, $\psi$ is a monomorphism. This fact is used to prove that the coalgebra $(H I, H \psi)$ is well-founded. Using an argument similar to Lambek's Lemma we derive that $\psi$ is invertible. Therefore results of [20] imply that the initial chain 
above converges, and for some ordinal $k, H^{k} 0$ is an initial algebra. Moreover, $H^{k} 0$ is by (a) a final well-founded coalgebra, thus, isomorphic to $\psi: I \longrightarrow H I$. Thus $\left(I, \psi^{-1}\right)$ is isomorphic to the initial algebra.

Theorem 2.14. For every endofunctor of Set we have:

initial algebra $=$ final well-founded coalgebra .

Proof (Sketch). There exists an endofunctor $H^{*}$ preserving finite intersections and agreeing on nonempty sets with $H$, see [19. Given $H$, we know from Theorem 2.13 that the equation above holds for $H^{*}$. From this one can prove it for $H$. The proof is quite technical because we need to compare well-foundedness of coalgebras for $H$ and $H^{*}$, and the empty set plays a substantial role here.

This last result and Corollary 2.12 serve as a basis for a description of initial algebras in Theorem 3.15 .

\section{Well-Pointed Coalgebras}

We arrive at the centerpiece of this paper, characterizations of the initial algebra, final coalgebra, and initial iterative algebra for set functors.

Throughout this section $H$ denotes an endofunctor of Set which preserves (wide) intersections. Many endofunctors of interest satisfy this condition, for example:

(a) the power-set functor, all polynomial functors, the finite distribution functor,

(b) products, coproducts, quotients, and subfunctors of functors preserving intersections, and

(c) "almost" all finitary functors: if $H$ is finitary then $H^{*}$ in Theorem 2.14 preserves intersections.

An example of a set functor not preserving intersections is the continuation monad $H X=R^{\left(R^{X}\right)}$, where $R$ is the set of results. A simpler example is the one taking every nonempty set to the terminal object and the empty set to itself.

By a pointed coalgebra is meant a triple $(A, a, x)$, where $(A, a)$ is a coalgebra and $x$ an element of $A$ called initial state. When speaking about morphisms between pointed coalgebras we mean those preserving the initial state. In particular, given a pointed coalgebra $1 \stackrel{x}{\longrightarrow} A \longrightarrow H A$ by a subobject is meant a subcoalgebra containing the initial state $x$.

Definition 3.1. A well-pointed coalgebra is a pointed coalgebra which has no proper subobjects and no proper quotients.

Remark 3.2. Recall that a simple coalgebra (called minimal coalgebra by Gumm[8]) is a coalgebra $(A, a)$ with no nontrivial quotient. That is, a coalgebra such that every homomorphism $h:(A, a) \longrightarrow(B, b)$ has $h$ monic. Gumm observed that 
(a) The full subcategory of Coalg $H$ given by all simple coalgebras is reflective: the reflection of a coalgebra $(A, a)$ is the simple quotient

$$
e_{(A, a)}:(A, a) \longrightarrow(\bar{A}, \bar{a})
$$

obtained as the wide pushout of all quotients of $(A, a)$.

(b) Every subcoalgebra of a simple coalgebra is simple.

(c) The coalgebra map $a: A \longrightarrow H A$ of a simple coalgebra is monic.

Remark 3.3. Thus, $1 \stackrel{x}{\longrightarrow} A \stackrel{a}{\longrightarrow} H A$ is a well-pointed coalgebra iff $(A, a)$ is simple and is generated by $x$. We call the latter condition reachability. That is, a pointed coalgebra is reachable if it has no proper pointed subcoalgebra. It is easy to see that this holds iff the canonical graph (see Definition 3.11 below) is reachable: every state has a directed path from the initial state.

Examples 3.4. (a) A deterministic automaton with a given initial state is a pointed coalgebra for $H X=X^{I} \times\{0,1\}$. Reachability means that every state can be reached (in finitely many steps) from the initial state. Simplicity means that the automaton is observable, i.e., for every pair of different states there exists an input word leading one of them to an accepting state and the other to a non-accepting state.

The usual terminology is that reachability and observability together are called minimality.

(b) For the power-set functor the pointed coalgebras are the pointed graphs. Well-pointed means reachable and simple, where simplicity states that no pair of different vertices is bisimilar.

Notation 3.5. Since $H$ preserves intersections, there is a canonical process of turning an arbitrary pointed coalgebra $(A, a, x)$ into a well-pointed one: form the simple quotient, see Remark 3.2 (a) pointed by $e_{(A, a)} \cdot x: 1 \longrightarrow \bar{A}$, then form the least subcoalgebra containing that point:

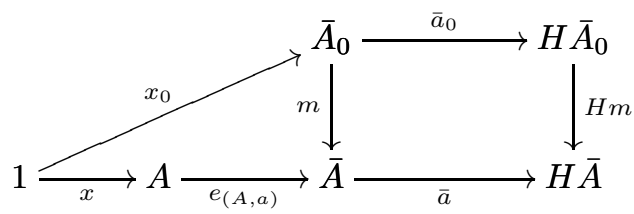

That is, $m$ is the intersection of all subcoalgebras of $(\bar{A}, \bar{a})$ through which $e_{(A, a)} \cdot x$ factorizes. Then $\left(\bar{A}_{0}, \bar{a}_{0}, x_{0}\right)$ is well-pointed due to Remark $[3.2(\mathrm{~b})$.

Example 3.6. For deterministic automata our process $A \longmapsto \bar{A}_{0}$ above means that we first merge the states that are observably equivalent and then discard the states that are not reachable. A more efficient way is first discarding the unreachable states and then merging observably equivalent pairs. Both ways are possible since our functor preserves inverse images: this implies that a quotient of a reachable pointed coalgebra is reachable. 
Notation 3.7. The collection of all well-pointed coalgebras up to isomorphism is denoted by

$$
\nu H \text {. }
$$

For every coalgebra $a: A \longrightarrow H A$ we have a function $a^{+}: A \longrightarrow \nu H$ assigning to every element $x: 1 \longrightarrow A$ the well-pointed coalgebra of Notation 3.5

$$
a^{+}(x)=\left(\bar{A}_{0}, \bar{a}_{0}, x_{0}\right) .
$$

Theorem 3.8. A set functor $H$ preserving intersections has a final coalgebra iff it has only a set of well-pointed coalgebras up to isomorphism. And, if it is the case, $\nu H$ is a final coalgebra.

Remark. Whenever $\nu H$ is a set, it carries a canonical coalgebra structure $\psi: \nu H$ $\longrightarrow H(\nu H)$. It assigns to every member $(A, a, x)$ of $\nu H$ the following element of $H(\nu H)$ :

$$
1 \stackrel{x}{\longrightarrow} A \stackrel{a}{\longrightarrow} H A \stackrel{H a^{+}}{\longrightarrow} H(\nu H) .
$$

We prove below that this is a final coalgebra.

Proof. (1) If $H$ has a final coalgebra, then due to Remark 3.2 every simple coalgebra is its subcoalgebra, since the unique homomorphism is monic. The final coalgebra has only a set of subcoalgebras, consequently, there exists up to isomorphism only a set of simple coalgebras. Consequently, only a set of wellpointed coalgebras.

(2) Let $H$ have a set $\nu H$ of representative well-pointed coalgebras. We prove that $\nu H$ with the coalgebra structure $\psi$ from (3.2) is final.

(2a) We first prove that for every coalgebra homomorphism $h:(A, a) \longrightarrow$ $(B, b)$ we have

$$
a^{+}=b^{+} \cdot h \text {. }
$$

Given $x: 1 \longrightarrow A$, then $b^{+} \cdot h$ assigns to it the well-pointed coalgebra $\left(\bar{B}_{0}, \bar{b}_{0}, y_{0}\right)$ obtained from $(B, b, y)$, where $y=h \cdot x$, as in Notation 3.5. It is not difficult, using Remark 3.2 , to prove that this well-pointed coalgebra is isomorphic to $\left(\bar{A}_{0}, \bar{a}_{0}, x\right)$.

(2b) $\nu H$ is a weakly final coalgebra because for every coalgebra $(A, a)$ we have a coalgebra homomorphism $a^{+}:(A, a) \longrightarrow(\nu H, x)$. Indeed, by 3.2 we have $\psi \cdot a^{+}(x)=H \bar{a}_{0}^{+} \cdot \bar{a}_{0}\left(x_{0}\right)$ and the diagram below shows that this is equal to $\mathrm{Ha}^{+} \cdot a(x)$ :

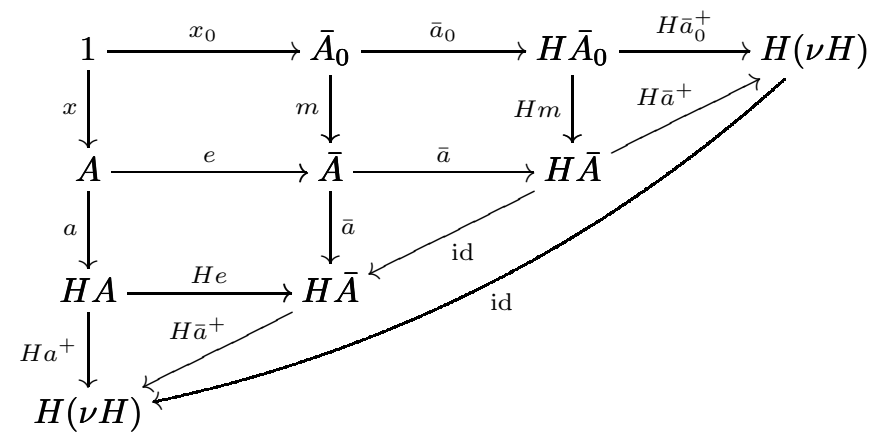


Notice that the upper and lower triangles commute since $m$ and $e$ are homomorphisms, see $(2 \mathrm{a})$.

(2c) We next prove that for the coalgebra $\psi: \nu H \longrightarrow H(\nu H)$ we have $\psi^{+}=\mathrm{id}_{\nu H}$. Indeed, given a well-pointed coalgebra $(A, a, x) \in \nu H$, consider the equality (3.3) with $h=a^{+}$(which is a homomorphism by $(2 \mathrm{~b})$ ) and $b=\psi$. Of course, $a^{+}(x)=(A, a, x)$, since $(A, a)$ is simple and $(A, a, x)$ is reachable. Then $\psi^{+}(A, a, x)=(A, a, x)$.

Finally, to prove uniqueness of the homomorphism $a^{+}$, suppose that $h:(A, a)$ $\longrightarrow(\nu H, \psi)$ is any homomorphism. Then we have

$$
a^{+} \stackrel{(2 a)}{=} \psi^{+} \cdot h \stackrel{(2 b)}{=} h \text {. }
$$

Examples 3.9. (a) For deterministic automata the final coalgebra (for $H X=$ $X^{I} \times\{0,1\}$ ) consists of all minimal (i. e., reachable and observable) automata. The more usual description is: the set $\mathscr{P} I^{*}$ of all formal languages. However, this is isomorphic: every formal language is accepted by a minimal automaton, unique up to isomorphism.

(b) The final coalgebra for the finite power-set functor is the coalgebra of all finitely branching well-pointed graphs. See Section 4 for more details.

Remark 3.10. If $\nu H$ is not a set, then $H$ does not have a (small) final coalgebra. However, $\nu H$ is its large final coalgebra: in the above proof smallness was not used.

Definition 3.11. For every coalgebra $a: A \longrightarrow H A$ define the canonical graph on $A$ : the neighbors of $x \in A$ are precisely those elements of $A$ which lie in the least subset $m: M \longleftrightarrow A$ with $a(x) \in H m[H M]$.

Proposition 3.12. A coalgebra for $H$ is well-founded iff its canonical graph is well-founded.

Remark. For functors $H$ preserving inverse images this fact is proved by Taylor, see 6.3.4 in [17. Our proof is essentially the same.

Corollary 3.13. Subcoalgebras of a well-founded coalgebra are well-founded.

Notation 3.14. The collection of all well-founded, well-pointed coalgebras (up to isomorphism) is denoted by

$$
\mu H \text {. }
$$

For every well-founded coalgebra $a: A \longrightarrow H A$ we have a function $a^{+}: A$ $\longrightarrow \mu H$ assigning to every element $x: 1 \longrightarrow A$ the well-founded, well-pointed coalgebra (3.1). Indeed, $\left(\bar{A}_{0}, \bar{a}_{0}\right)$ is well-founded due to Corollaries 2.12 and 3.13 .

Theorem 3.15. A set functor $H$ preservring intersections has an initial algebra iff it has only a set of well-founded, well-pointed coalgebras up to isomorphism. And, if it is the case, $\mu \mathrm{H}$ is an initial algebra. 
Remark. Whenever $\mu H$ is a set, it carries a canonical coalgebra structure $\bar{\psi}: \mu H$ $\longrightarrow H(\mu H)$ defined by (3.2). We prove below that this is a final well-founded coalgebra. Thus, by Theorem 2.14, $\mu H$ is an initial algebra with the structure given by the inverse of $\bar{\psi}$.

Proof. (1) If $H$ has an initial algebra $I$, then by Theorem 2.14 this is a final wellfounded coalgebra. Every well-founded, well-pointed coalgebra is simple, whence a subcoalgebra of $I$ since the unique homomorphism into $I$ is monomorphic by Remark 3.2. Consequently, $\mu H$ is a set.

(2) Let $H$ have a set $\mu H$ of representatives of well-founded, well-pointed coalgebras. The proof that for every well-founded coalgebra $(A, a)$ the map $a^{+}: A$ $\longrightarrow \mu H$ is a unique coalgebra homomorphism into $\bar{\psi}: \mu H \longrightarrow H(\mu H)$ is completely analogous to the proof of finality of $\psi: \nu H \longrightarrow H(\nu H)$ in Theorem 3.8 Just recall that subcoalgebras and quotients of a well-founded coalgebra are all well-founded (by Corollaries 2.12 and 3.13 ).

It remains to prove that $(\mu H, \bar{\psi})$ is a well-founded coalgebra. To this end notice that for every well-pointed, well-founded coalgebra $(A, a, x)$ in $\mu H$ we have $a^{+}(x)=(A, a, x)$. Now take the coproduct (in Coalg $\left.H\right)$ of all $(A, a)$ for which there is an $x \in A$ such that $(A, a, x)$ lies in $\mu H$. This coproduct is a well-founded coalgebra by Corollary 2.12, and, as we have just seen, the unique induced homomorphism from the coproduct into $(\mu H, \bar{\psi})$ is epimorphic, whence $\mu H$ is a quotient coalgebra of the coproduct. Thus, another application of Corollary 2.12 shows that $\mu H$ is a well-founded coalgebra as desired.

Remark 3.16. (a) Recall from [4 that an algebra $a: H A \longrightarrow A$ is iterative provided that every (equation) morphism $e: X \longrightarrow H X+A$, where $X$ is a finite set, has a unique solution, i.e., $e^{\dagger}: X \longrightarrow A$ such that $e^{\dagger}=[a, A] \cdot\left(H e^{\dagger}+A\right) \cdot e$. It was proved in 10 that the initial iterative algebra is precisely the final locally finite coalgebra, where a coalgebra is called locally finite if every element of it lies in a finite subcoalgebra.

Example 3.17 (see [4]). The initial iterative algebra for $H X=X^{I} \times\{0,1\}$ consists of all finite minimal automata. This is isomorphic to its description as all regular languages.

Notation 3.18. For every finitary set functor denote by

$$
\varrho H
$$

the set of all finite well-pointed coalgebras up to isomorphism.

Given a finite coalgebra $a: A \longrightarrow H A$ we define a function $a^{+}: A \longrightarrow \varrho H$ by (3.1).

Theorem 3.19. Every finitary set functor $H$ has an initial iterative algebra $\varrho H$ formed by all finite well-pointed coalgebras.

Remark. $\varrho H$ has the canonical coalgebra structure $\tilde{\psi}: \varrho H \longrightarrow H(\varrho H)$ given by (3.2). The proof that this is the final locally finite coalgebra is analogous to the proof of Theorem 3.8 . 


\section{Examples of Well-Pointed Coalgebras}

Example 4.1. Deterministic automata, $H X=X^{I} \times\{0,1\}$. In Example 3.9 we saw that $\nu H$ consists of all minimal automata, or, equivalently, all languages over $I$. The initial iterative algebra $\varrho H$ consists of all finite minimal automata, this is isomorphic to

$$
\varrho H=\text { all regular languages. }
$$

Finally, no well-pointed coalgebra is well-founded because the empty subcoalgebra is cartesian, thus,

$$
\mu H=\emptyset .
$$

Example 4.2. Streams. Consider the coalgebras for $H X=X \times I+1$. Jan Rutten [13] interprets them as dynamical systems with outputs in $I$ and with terminating states (where no next state is given). Every state $q$ yields a stream, finite or infinite, over $I$ by starting in $q$ and traversing the dynamic system as long as possible. We call it the response of $q$. It is an element of $I^{\omega}+I^{*}$.

(a) For every word $s_{1} \cdots s_{n}$ in $I^{*}$ we have a well-pointed dynamic system

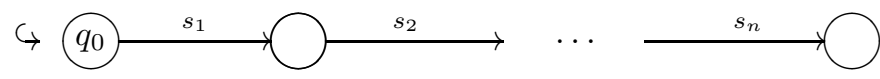

(b) For every eventually periodic stream in $I^{\omega}$,

$$
w=u v^{\omega} \quad \text { for } u, v \in I^{*},
$$

we have a well-pointed dynamic system which uses $u$ as in (a) and adds a cycle repeating $v$.

The following was already proved by Arbib and Manes [9, 10.2.5].

Corollary 4.3. For $H X=X \times I+1$ we have

$$
\begin{aligned}
& \nu H \cong I^{*}+I^{\omega}, \quad \text { all finite and infinite streams, } \\
& \varrho H \cong \text { all finite and eventually periodic streams, } \\
& \mu H \cong I^{*}, \quad \text { all finite streams. }
\end{aligned}
$$

Example 4.4. Binary trees. Coalgebras for the functor

$$
H X=X \times X+1
$$

are given, as observed by Jan Rutten [13, by a set $Q$ of states which are either terminating or have precisely two next states according to a binary input, say $\{l, r\}$. Every state $q \in Q$ yields an ordered binary tree $T_{q}$ (i.e, nodes that are not leaves have a left-hand child and a right-hand one) by tree expansion: the root is $q$ and a node is either a leaf, if it is a terminating state, or has the two next states as children (left-hand for input $l$, right-hand for input $r$ ). Binary trees are considered up to isomorphism. 
Lemma 4.5. For every coalgebra of the functor $H X=X \times X+1$ the largest congruence merges precisely the pairs of states having the same tree expansion.

Proof. Let $\sim$ be the equivalence with $q \sim q^{\prime}$ iff $T_{q}=T_{q^{\prime}}$. There is an obvious structure of a coalgebra on $Q / \sim$ showing that $\sim$ is a congruence. For every coalgebra homomorphism $h: Q \longrightarrow \bar{Q}$ the tree expansion of $q \in Q$ is always the same as the tree expansion of $h(q)$ in $\bar{Q}$. Thus, $\sim$ is the largest congruence.

Corollary 4.6. A well-pointed coalgebra of the functor $H X=X \times X+1$ is a coalgebra with an initial state $q_{0}$ which is reachable (every state can be reached from $q_{0}$ ) and simple (different states have different tree expansions).

Moreover, tree expansion of the initial state is a bijection between well-pointed coalgebras and binary trees. The coalgebra is finite iff the tree expansion is rational, i.e., it has only finitely many subtrees up to isomorphism. And the well-founded coalgebras are precisely those yielding a finite tree expansion.

The following result was proved by Arbib and Manes [9], 10.2.5 (description of $\nu H)$ and in [4] (description of $\rho H$ ).

Corollary 4.7. For the functor $H X=X \times X+1$ we have

$$
\begin{aligned}
& \nu H \cong \text { all binary trees } \\
& \varrho H \cong \text { all rational binary trees } \\
& \mu H \cong \text { all finite binary trees. }
\end{aligned}
$$

Example 4.8. Graphs. Here we investigate coalgebras for the power-set functor $\mathscr{P}$ (that is, graphs) and for the finitary power-set functor $\mathscr{P}_{\omega}$ (that is, finitely branching graphs). In the rest of Section 4 all trees are understood to be non-ordered. That is, a tree is a directed graph with a node (root) from which every node can be reached by a unique path.

Recall the concept of a bisimulation between graphs $X$ and $Y$ : it is a relation $R \subseteq X \times Y$ such that whenever $x R y$ then every child of $x$ is related to a child of $y$, and vice versa. Two nodes of a graph $X$ are called bisimilar if they are related by a bisimulation $R \subseteq X \times X$.

Lemma 4.9. The greatest congruence on a graph merges precisely the bisimilar pairs of states.

This follows, since $\mathscr{P}$ preserves weak pullbacks, from general results of Rutten [13].

Corollary 4.10. A pointed graph $\left(G, q_{0}\right)$ is well-pointed iff it is reachable (every vertex can be reached from $q_{0}$ by a directed path) and simple (all distinct pairs of states are non-bisimilar).

Example 4.11. Peter Aczel introduced in [1] the canonical picture of a (wellfounded) set $X$. It is the graph with vertices all sets $Y$ such that a sequence

$$
Y=Y_{0} \in Y_{1} \in \cdots \in Y_{n}=X
$$


of sets exists. The neighbors of a vertex $Y$ are all of its elements. When pointed by $X$, this is a well-pointed graph which is, due to the Foundation Axiom, wellfounded. Conversely, every well-founded well-pointed graph is isomorphic to the canonical picture of a set.

Corollary 4.12. $\mu \mathscr{P}=$ all sets.

This was proved by Rutten and Turi in [14. The bijection between well-founded, well-pointed graphs and sets (given by the canonical picture) takes the finite well-founded graphs to the hereditarily finite sets $X$, i.e., finite sets with finite elements which also have finite elements, etc. More precisely: a set is hereditarily finite if all sets in the canonical picture of $X$ are finite:

Corollary 4.13. $\mu \mathscr{P}_{\omega}=$ all hereditarily finite sets.

In order to describe the final coalgebra for $\mathscr{P}$ in a similar set-theoretic manner, we must move from the classical theory to the non-well-founded set theory of Peter Aczel [1]. Recall that a decoration of a graph is a coalgebra homomorphism from this graph into the large coalgebra $($ Set,$\in$ ). Non-well-founded set theory is obtained by swapping the axiom of foundation, telling us that $($ Set, $\in$ ) is well-founded, with the following

Anti-Foundation Axiom. Every graph has a unique decoration.

Example 4.14. The decoration of a single loop is a set $\Omega$ such that $\Omega=\{\Omega\}$.

The coalgebra (Set, $\in$ ) where now Set is the class of all non-well-founded sets, is of course final: the decoration of $G$ is the unique homomorphism $d: G$ Set.

Corollary 4.15. In the non-well-founded set theory: $\nu \mathscr{P}=$ all sets.

Let us turn to the finite power-set functor $\mathscr{P}_{\omega}$.

Remark 4.16. Worrell introduced in [21] the notion of a tree-bisimulation between trees $T_{1}$ and $T_{2}$; this is a graph bisimulation $R \subseteq T_{1} \times T_{2}$ which relates the roots and such that $x_{1} R x_{2}$ implies that $x_{1}$ and $x_{2}$ are the roots or have related parents.

A tree $T$ is called strongly extensional iff every tree bisimulation $R \subseteq T \times T$ is trivial: $R \subseteq \Delta_{T}$. The tree expansion is a bijection between all well-pointed finitely branching graphs and strongly extensional finitely branching trees.

Corollary 4.17. For the finite power-set functor $\mathscr{P}_{\omega}$ we have

$\nu \mathscr{P}_{\omega}=$ all finitely branching, strongly extensional trees, $\varrho \mathscr{P}_{\omega}=$ all finitely branching, rational, strongly extensional trees, $\mu \mathscr{P}_{\omega}=$ all finite strongly extensional trees. 
Example 4.18. Labeled transition systems. Here we consider, for a set $A$ of actions, coalgebras for $\mathscr{P}_{\omega}(-\times A)$. A bisimulation between two finitely branching labeled transition systems (LTS) $G$ and $G^{\prime}$ is a relation $R \subseteq G \times G^{\prime}$ such that

if $\quad x R y$ then for every transition $x \stackrel{a}{\longrightarrow} x^{\prime}$ in $G$ there exists a transition $y \stackrel{a}{\longrightarrow} y^{\prime}$ with $x^{\prime} R y^{\prime}$, and vice versa.

States $x, y$ of an LTS are called bisimilar if $x R y$ for some bisimulation $R \subseteq$ $G \times G$.

A well-pointed LTS is an LTS together with an initial state $q_{0}$ which is reachable (every state can be reached from $q_{0}$ ) and simple (distinct states are nonbisimilar).

The tree expansion of a state $q$ is a (non-ordered) tree with edges labeled in $A$, shortly, an $A$-labeled tree. For $A$-labeled trees we modify Definition 4.16 in an obvious manner.

Corollary 4.19. For the finitely branching LTS we have

$$
\begin{aligned}
\nu \mathscr{P}_{\omega}(-\times A)= & \text { all finitely branching, strongly extensional } A \text {-labeled trees, } \\
\varrho \mathscr{P}_{\omega}(-\times A)= & \text { all rational, finitely branching, strongly extensional } \\
& \text { A-labeled trees, } \\
\mu \mathscr{P}_{\omega}(-\times A)= & \text { all finite extensional } A \text {-labeled trees. }
\end{aligned}
$$

\section{Conclusions}

For set functors $H$ satisfying the (mild) assumption of preservation of intersections we described (a) the final coalgebra as the set of all well-pointed coalgebras, (b) the initial algebra as the set of all well-pointed coalgebras that are well-founded, and (c) in the case where $H$ is finitary, the initial iterative algebra as the set of all finite well-pointed coalgebras. This is based on the observation that given an element of a final coalgebra, the subcoalgebra it generates has no proper subcoalgebras nor proper quotients - shortly, this subcoalgebra is well-pointed. And different elements define nonisomorphic well-pointed subcoalgebras. We then combined this with our result that for all set functors the initial algebra is precisely the final well-founded coalgebra. This resulted in the above description of the initial algebra. Numerous examples demonstrate that this view of final coalgebras and initial algebras is useful in applications.

Whereas our result about well-founded coalgebras was proved in locally finitely presentable categories, the description of the final coalgebra was formulated for set functors only. In future research we intend to generalize this result to a wider class of base categories. 


\section{References}

1. Aczel, P.: Non-well-founded Sets. CSLL Lect. Notes, vol. 14. Stanford CSLI Publications, Stanford (1988)

2. Adámek, J.: Free algebras and automata realizations in the language of categories. Comment. Math. Univ. Carolinæ 15, 589-602 (1974)

3. Adámek, J., Herrlich, H., Strecker, G.E.: Abstract and Concrete Categories. John Wiley and Sons, New York (1990)

4. Adámek, J., Milius, S., Velebil, J.: Iterative algebras at work. Math. Structures Comput. Sci. 16, 1085-1131 (2006)

5. Adámek, J., Rosický, J.: Locally Presentable and Accessible Categories. Cambridge University Press (1994)

6. Capretta, V., Uustalu, T., Vene, V.: Recursive coalgebras from comonads. Inform. and Comput. 204, 437-468 (2006)

7. Gabriel, P., Ulmer, F.: Lokal präsentierbare Kategorien. Lecture Notes in Math., vol. 221. Springer, Berlin (1971)

8. Gumm, H.-P.: On minimal coalgebras. Appl. Categ. Structures 16, 313-332 (2008)

9. Manes, E.G., Arbib, M.A.: Algebraic Approaches to Program Semantics. Springer, New York (1986)

10. Milius, S.: A sound and complete calculus for finite stream circuits. In: Proc. 25th Annual Symposium on Logic in Computer Science (LICS 2010), pp. 449-458. IEEE Computer Society (2010)

11. Nelson, E.: Iterative algebras. Theoret. Comput. Sci. 25, 67-94 (1983)

12. Osius, G.: Categorical set theory: a characterization of the category of sets. J. Pure Appl. Algebra 4, 79-119 (1974)

13. Rutten, J.J.M.M.: Universal coalgebra: a theory of systems. Theoret. Comput. Sci. 249, 3-80 (2000)

14. Rutten, J.J.M.M., Turi, D.: On the Foundations of Final Semantics: Non-Standard Sets, Metric Spaces, Partial Orders. In: de Bakker, J.W., de Roever, W.-P., Rozenberg, G. (eds.) REX 1992. LNCS, vol. 666, pp. 477-530. Springer, Heidelberg (1993)

15. Tarski, A.: A lattice theoretical fixed point theorem and its applications. Pacific J. Math. 5, 285-309 (1955)

16. Taylor, P.: Towards a unified treatement of induction I: the general recursion theorem, preprint (1995-6), http://www.paultaylor.eu/ordinals/\#towuti

17. Taylor, P.: Practical Foundations of Mathematics. Cambridge University Press (1999)

18. Tiurin, J.: Unique fixed points vs. least fixed points. Theoret. Comput. Sci. 12, 229-254 (1980)

19. Trnková, V.: On a descriptive classification of set functor I. Comment. Math. Univ. Carolinæ 12, 323-352 (1971)

20. Trnková, V., Adámek, J., Koubek, V., Reiterman, J.: Free algebras, input processes and free monads. Comment. Math. Univ. Carolinæ 16, 339-351 (1975)

21. Worrell, J.: On the final sequence of a finitary set functor. Theoret. Comput. Sci. 338, 184-199 (2005) 